\title{
Inclusion of Body-Bias Effect in SPICE Modeling of 4H-SiC Integrated Circuit Resistors
}

\author{
Philip G. Neudeck, NASA Glenn Research Center, Cleveland, OH USA, Neudeck@nasa.gov, https://sic.grc.nasa.gov
}

Abstract. The direct-current (DC) electrical behavior of $n$-type $4 \mathrm{H}$-SiC resistors used for realizing $500{ }^{\circ} \mathrm{C}$ durable integrated circuits (ICS) is studied as a function of substrate bias and temperature. Improved fidelity electrical simulation is described using the SPICE NMOS model to simulate resistor substrate body-bias effect which is absent from the SPICE semiconductor resistor model.

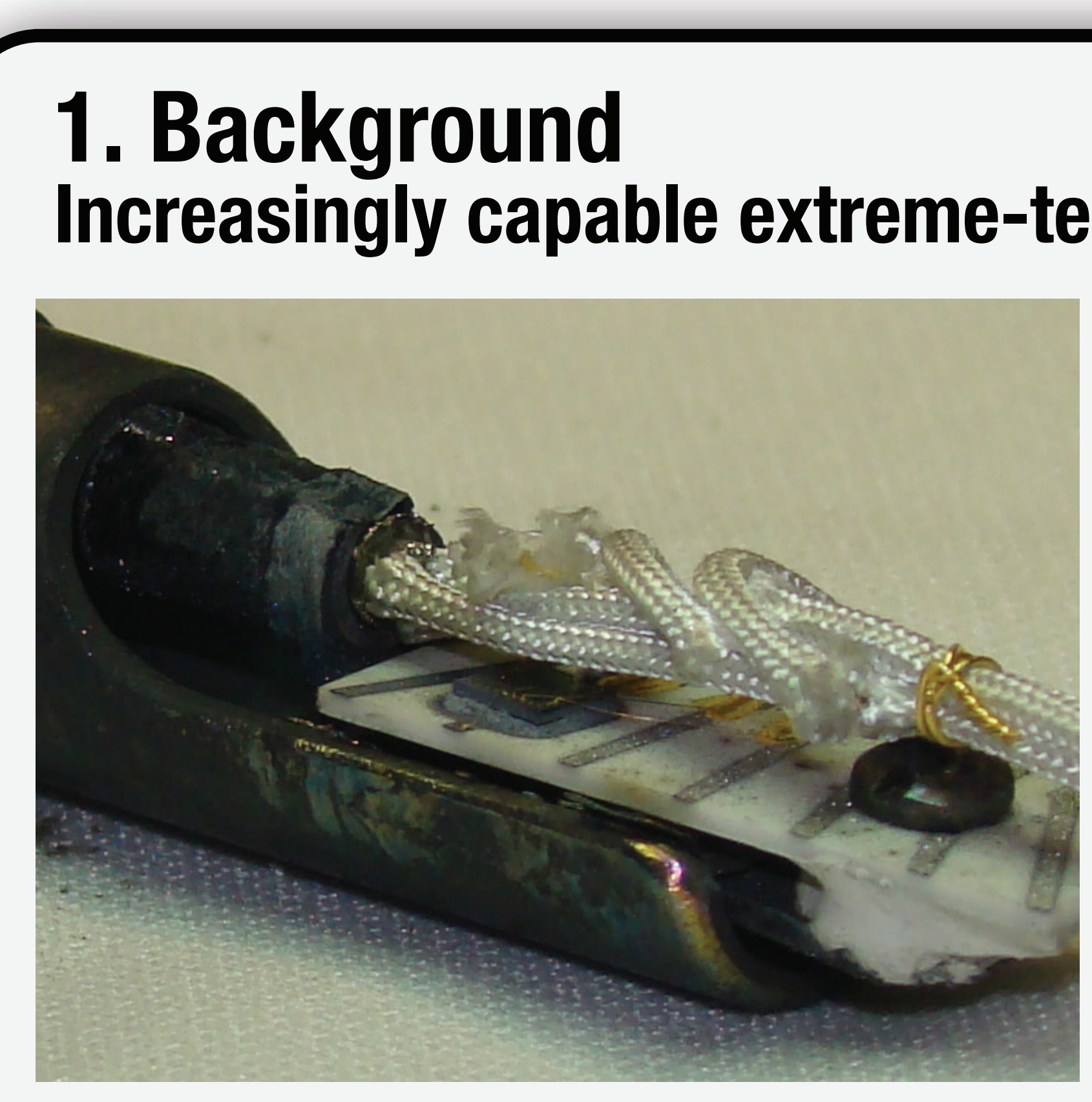

Fig. 1: 3mm-by-3mm SiC JFET ring oscillator chip that operated for 3 weeks exposed to $460^{\circ} \mathrm{C} 9.4 \mathrm{MPa}$ caustic Venus surface atmospheric conditions [1]

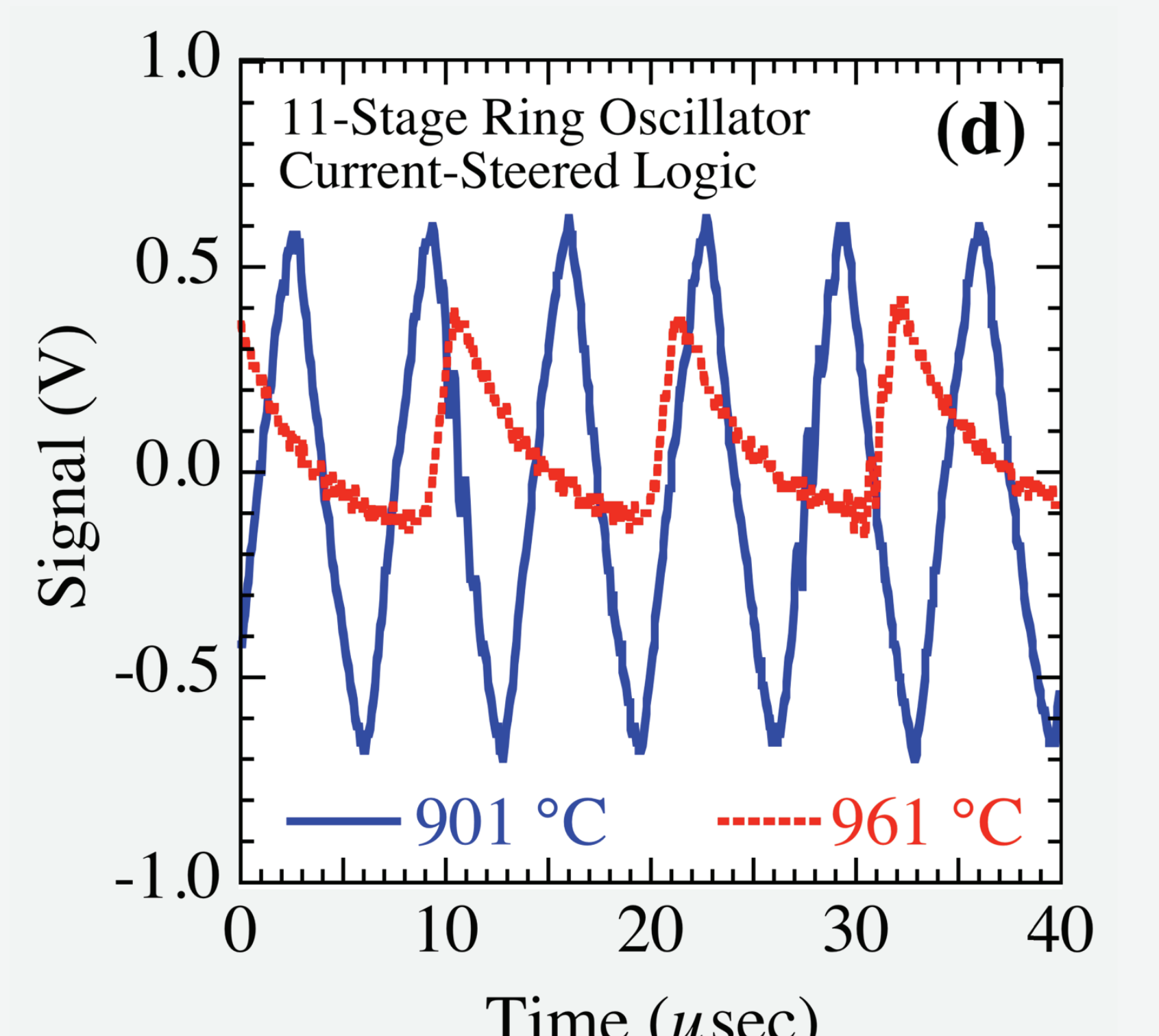

Fig. 2: SiC JFET ring oscillator waveforms at record high temperature $>900^{\circ} \mathrm{C}[2]$.

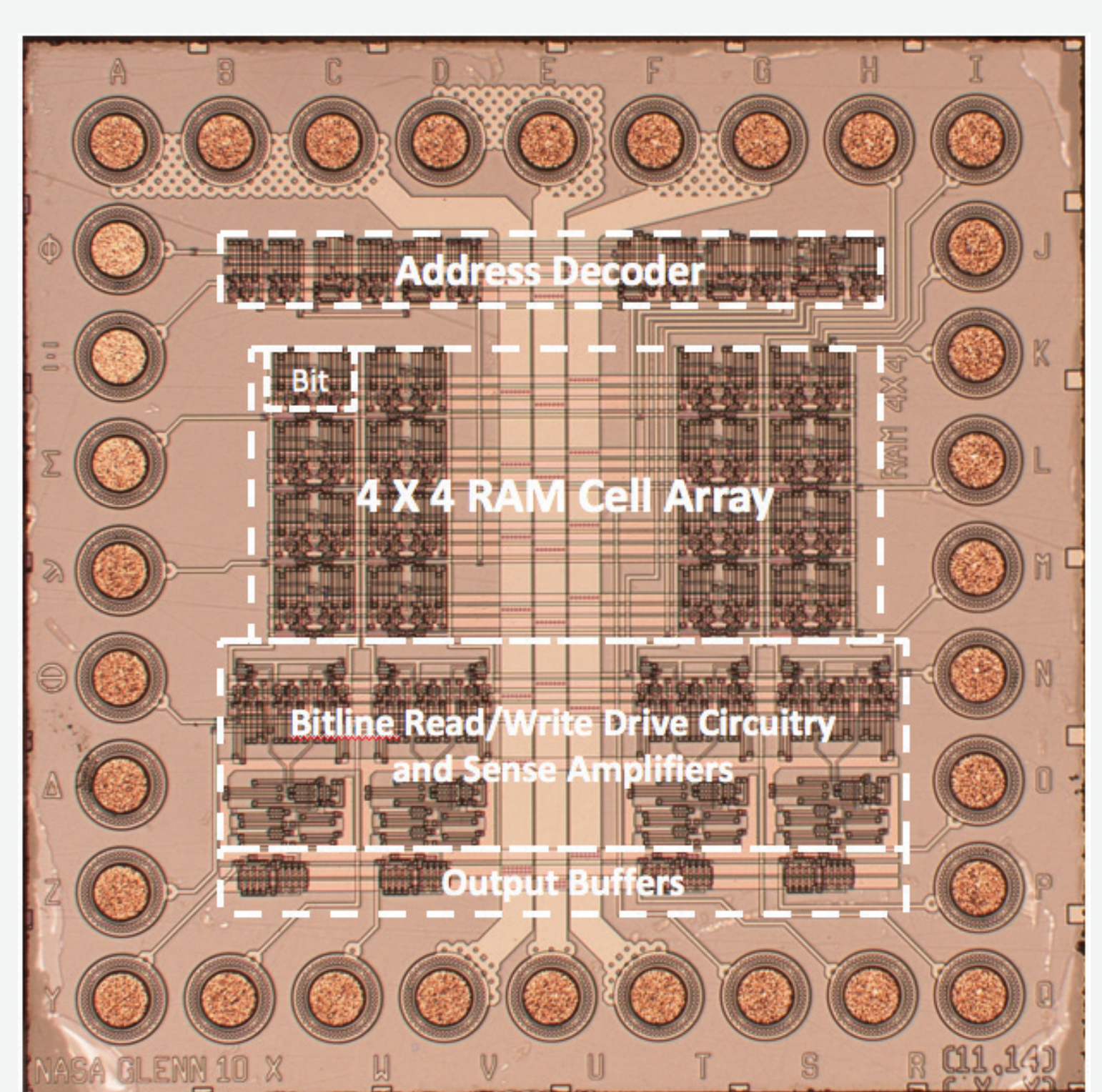

Fig. 3: 3mm-by-3mm SiC RAM chip (195 JFETs) operational for 5000 hours at $500^{\circ} \mathrm{C}$ [FR.D1.4 by D. Spry, Friday 9:30 AM].
Circuit engineers need SPICE models to design application-specific 4H-SiC JFET ICs.

\section{Body-Bias Effect}

As shown below in Fig. 4, the cross-sectional illustration of the SiC resistor structure used to realize the above JFET IC's, bias-dependent substrate pn junction depletion affects undepleted thickness (and therefore conductivity) of the resistor n-channel.

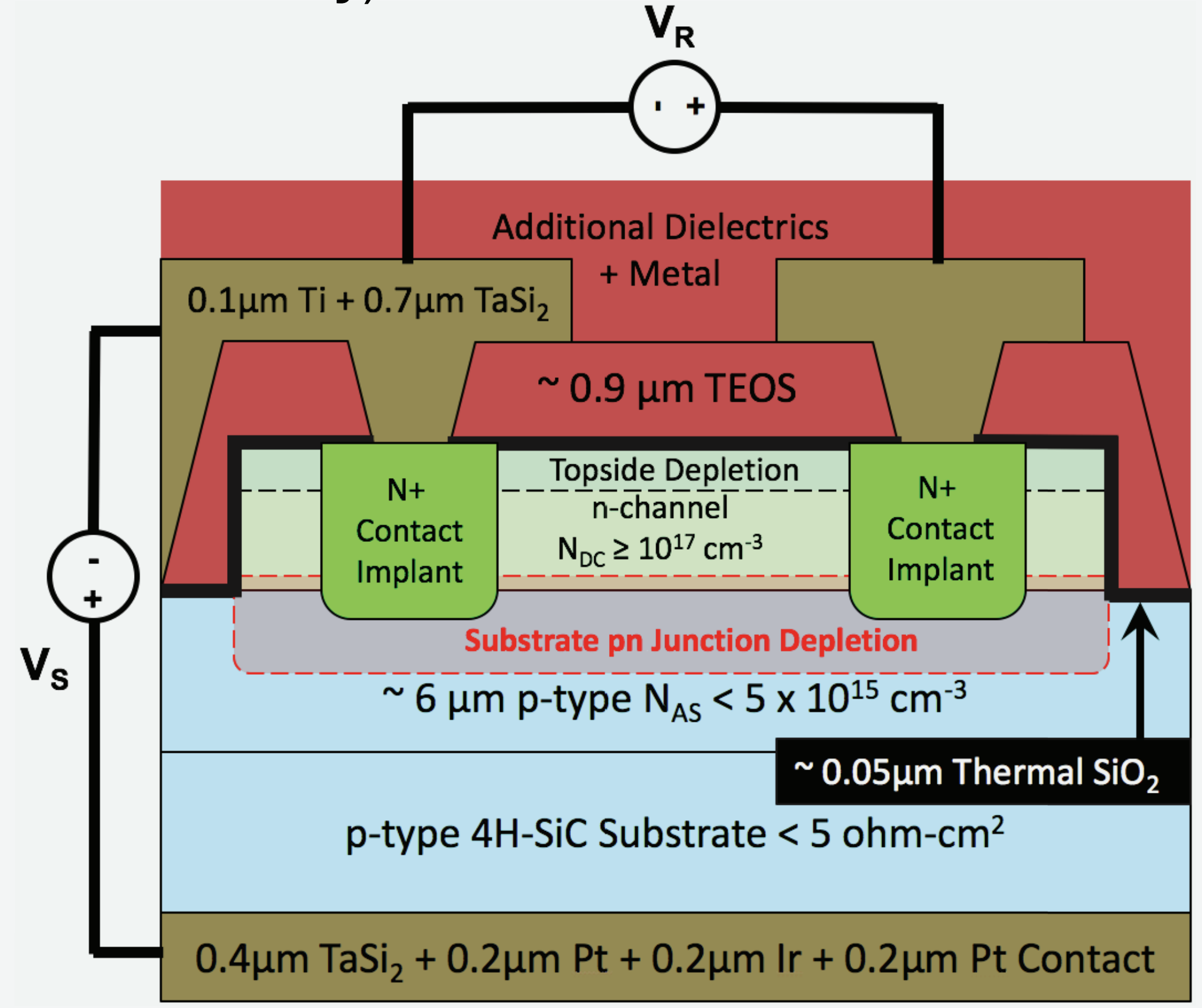

Experimental SiC fabrication, packaging, and measurement setup are described in [2-5]

For applied resistor bias $V_{R}>0$, depletion widths change across the lateral length of the resistor.

Current vs. voltage (I-V) characteristics and the IC resistor resistance value depend on both $V_{R}$ and $V_{S}$

Fig. 4: Cross-sectional illustration of SiC resistor structure used in JFET IC's, including substrate pn junction depletion that gives rise to resistor body bias effect and applied substrate bias voltage $V_{s}$ and resistor bias voltage $V_{R}$

\section{Measured Impact of Body Bias Effect on Resistor I-V Characteristics}

Body-bias effect causes mild non-linearity (i.e., downward bending) of resistor I-V characteristics (shown in green).

Amount of non-linearity can be quantified by plotting differential resistance $R_{\mathrm{D} u=}=\mathrm{d} / \mathrm{d} \mathrm{V}$ (shown in blue).
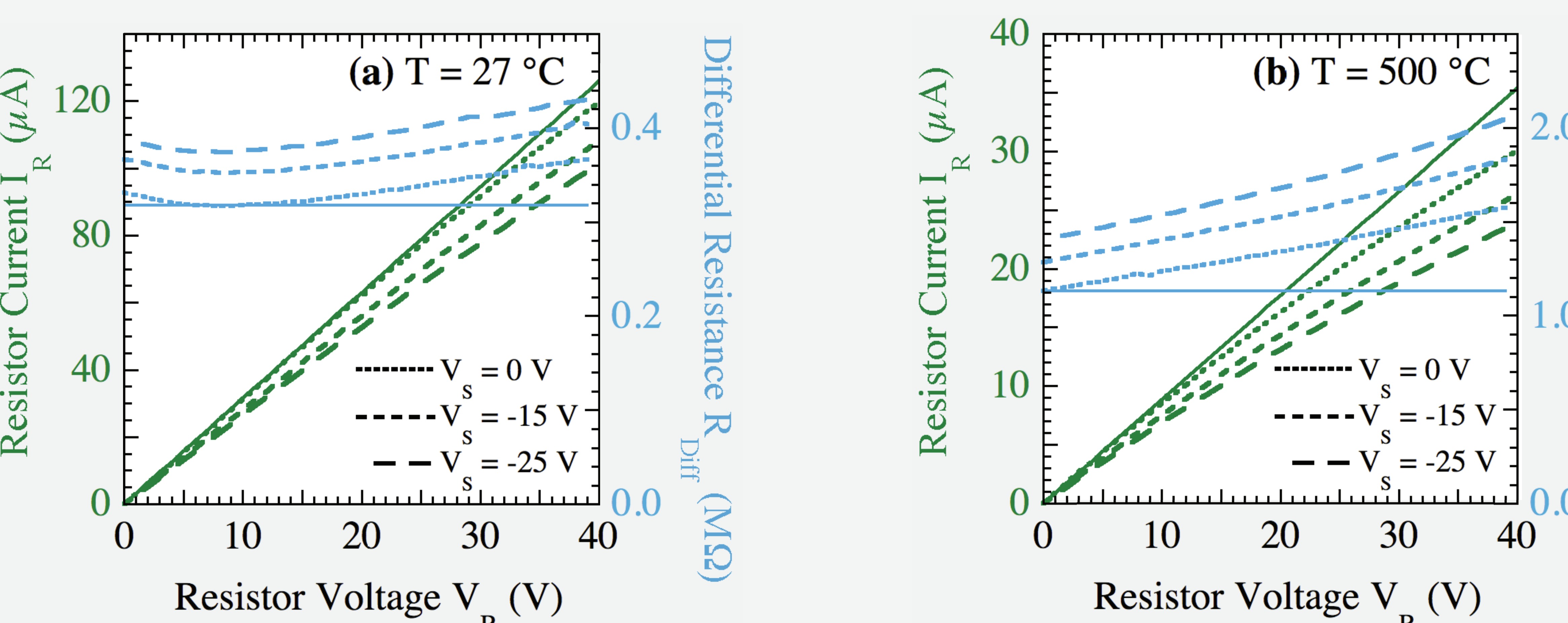

Fig. 5: Comparisons of DC-measured 80-square resistor I-V characteristics (dashed lines) with SPICE resistor mode simulations (solid lines) at $V_{s}$ of $0,-15$, and $-25 \mathrm{~V}$ at (a) $27^{\circ} \mathrm{C}$ and (b) $500{ }^{\circ} \mathrm{C}$.

Baseline SPICE resistor model (solid lines) [6] does not account for substrate body-bias effect, so it is incapable of modeling experimentally observed JFET IC resistors (and I-V characteristics) with high degree of accuracy.

Fails to model observed I-V bending/nonlinearity with increasing resistor bias $V_{\text {. }}$

Fails to model observed dependence on substrate bias $V$

Slightly rectifying metal contacts at $27^{\circ} \mathrm{C}$ impacts

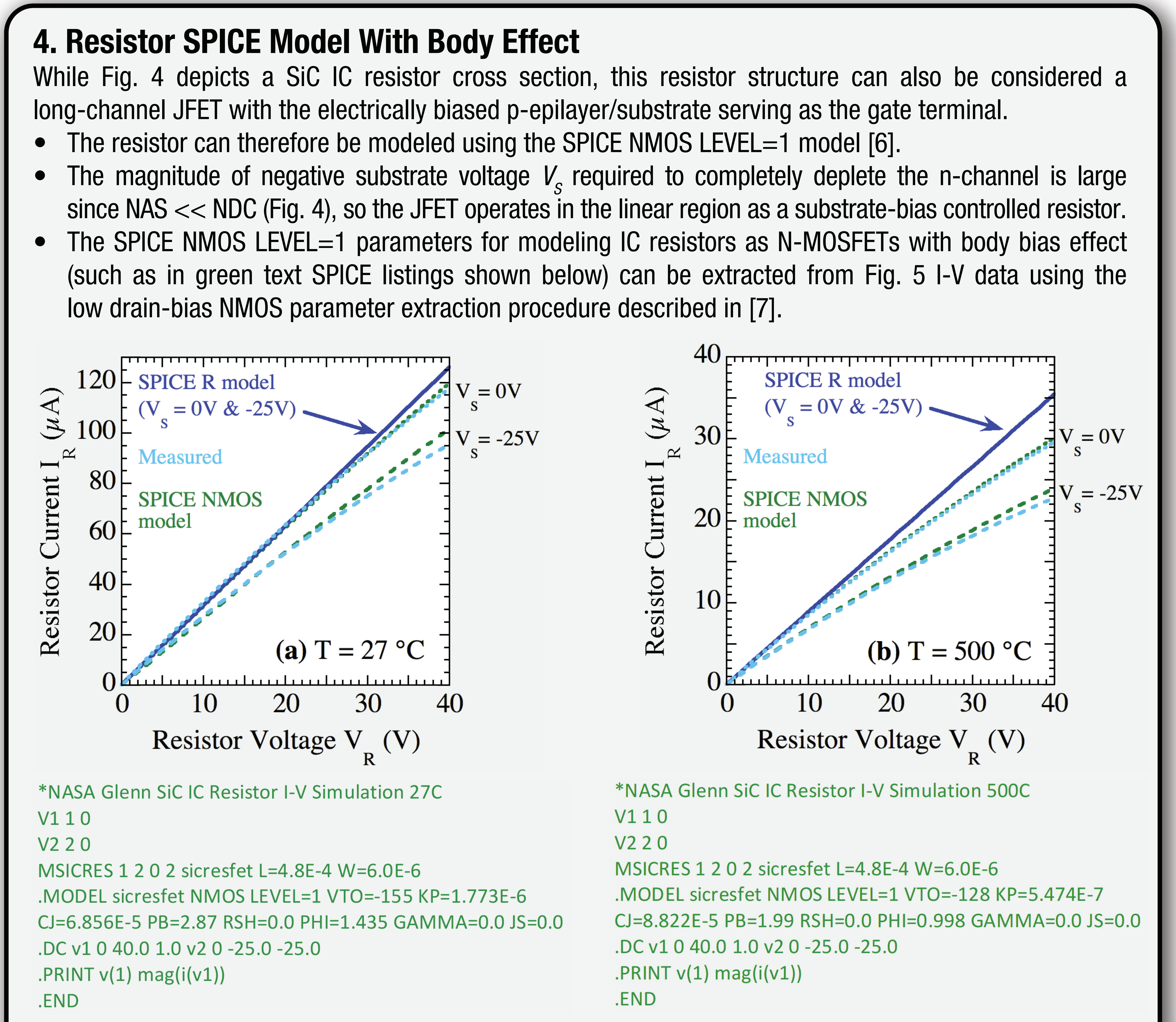

Fig. 6: I-V comparison of linear R SPICE resistor model (solid dark blue) and NMOS SPICE body-effect resistor model (dashed green) with measured data (dashed light blue) for a 480 -by-6um resistor at (a) $27^{\circ} \mathrm{C}$ and (b) $500{ }^{\circ} \mathrm{C}$. The SPICE decks for the NMOS model simulations are shown in green text below each plot.

Substantially improved agreement with measured $I-V$ is obtained using the NMOS SPICE model I-V compared with the SPICE R model I-V.

\section{Conclusion}

For improved accuracy circuit design and modeling of 4H-SiC JFET ICs using SPICE, the NMOS resistor modeling approach described in this report should supercede/replace the standard SPICE R resistor model reported in [6], which ignores the body-bias effect.

\section{Future Work}

Comparison and design studies of SiC JFET integrated circuits using new SPICE NMOS resistor modeling

\section{Acknowledgements}

D. Spry, L. Chen, G. Beheim, N. Prokop, M. Krasowski, K. Moses, J. Gonzalez, M. Mrdenovich, R. Buttler, R. Meredith, D. Lukco, C. Chang, G. Hunter, G. Ponchak, L. Matus. Work funded jointly by NASA Aeronautics (Transformative Tools and Technologies project) and Science Mission (Planetary Instrument Concepts for the Advancement of Solar System Observations project) Directorates.

\section{References}

[1] P. G. Neudeck et al., AIP Advances 6 (2016) 125119

[2] P. G. Neudeck et al., IEEE Electron Device Lett. 38 (2017) 1082-1085.

3] D.J. Spry, et al., IEEE Electron Device Lett. 37 (2016) 625-628.

[4] D.J. Spry, et al., Proc. IMAPS Int. High Temperature Electronics Conf. (2016) 249-256.

[5] L. Chen, et al., Proc. IMAPS Int. High Temperature Electronics Conf. (2016) 66-72.

[6] P. G. Neudeck, et al., Proc. IMAPS Int. High Temperature Electronics Conf. (2016) 263-271. Available https://sic.grc.nasa.gov/files/HiTEC2016-SPICEPaperV6.pdf

7] E. Profumo, MOS Parameter Measurements, in: P. Antognetti, G. Massobrio (Eds.), Semiconductor Device Modeling with SPICE, McGraw-Hill, New York, 1987, pp. 231-234. 